\title{
DUNKL-WILLIAMS INEQUALITIES FOR INTEGRABLE FUNCTIONS IN BANACH SPACE
}

\author{
JIANBING CAO
}

(Received 8 April 2012; accepted 13 September 2012; first published online 12 December 2012)

\begin{abstract}
In this paper, a generalisation of the Dunkl-Williams inequality is established for strongly integrable functions with values in a Banach space. Some applications are also presented.

2010 Mathematics subject classification: primary 26D15; secondary 46B20, 46B99.

Keywords and phrases: Dunkl-Williams inequality, triangle inequality, Banach space.
\end{abstract}

\section{Introduction and preliminaries}

The well-known Dunkl-Williams inequality [4] states that for any two nonzero elements $x, y$ in a normed linear space

$$
\left\|\frac{x}{\|x\|}-\frac{y}{\|y\|}\right\| \leq \frac{4\|x-y\|}{\|x\|+\|y\|}
$$

Over the years, various refinements of (1.1) have been obtained (see, for example, $[1,2,7,8,11])$. In [10], the authors considered the case of equality in some generalisations of the Dunkl-Williams inequality for elements of a pre-Hilbert $C^{*}$ module. Recently, Pečarič et al. [9] presented the following general Dunkl-Williams inequality for an arbitrary number of finitely many nonzero elements of a normed linear space:

$$
\begin{aligned}
& \left\|\sum_{j=1}^{n} \frac{x_{j}}{\left\|x_{j}\right\|}\right\| \leq \min _{1 \leq i \leq n}\left\{\frac{1}{\left\|x_{i}\right\|}\left(\left\|\sum_{j=1}^{n} x_{j}\right\|+\sum_{j=1}^{n} \mid\left\|x_{j}\right\|-\left\|x_{i}\right\|\right)\right\}, \\
& \left\|\sum_{j=1}^{n} \frac{x_{j}}{\left\|x_{j}\right\|}\right\| \geq \max _{1 \leq i \leq n}\left\{\frac{1}{\left\|x_{i}\right\|}\left(\left\|\sum_{j=1}^{n} x_{j}\right\|-\sum_{j=1}^{n}\left|\left\|x_{j}\right\|-\left\|x_{i}\right\|\right|\right)\right\} .
\end{aligned}
$$

They also considered conditions for equality in (1.2) and (1.3) to hold in strictly convex normed linear space. In [9], the authors showed that these inequalities imply 
the following refinements of the generalised triangle inequalities obtained by Kato et al. in [6]:

$$
\begin{aligned}
& \sum_{j=1}^{n}\left\|x_{j}\right\| \geq\left\|\sum_{j=1}^{n} x_{j}\right\|+\left(n-\left\|\sum_{j=1}^{n} \frac{x_{j}}{\left\|x_{j}\right\|}\right\|\right) \min _{1 \leq j \leq n}\left\{\left\|x_{j}\right\|\right\}, \\
& \sum_{j=1}^{n}\left\|x_{j}\right\| \leq\left\|\sum_{j=1}^{n} x_{j}\right\|+\left(n-\left\|\sum_{j=1}^{n} \frac{x_{j}}{\left\|x_{j}\right\|}\right\|\right) \max _{1 \leq j \leq n}\left\{\left\|x_{j}\right\|\right\} .
\end{aligned}
$$

Recently, continuous versions of (1.4) and (1.5) have been obtained by Hsu et al. in [5]:

$$
\begin{aligned}
& \int_{\Omega} a(t)\|f(t)\| d \mu \geq\left\|\int_{\Omega} a(t) f(t) d \mu\right\|+\left(\|a\|_{1}-\left\|\int_{\Omega} \frac{a(t) f(t)}{\|f(t)\|} d \mu\right\|\right) \operatorname{ess} \inf (\|f(\cdot)\|), \\
& \int_{\Omega} a(t)\|f(t)\| d \mu \leq\left\|\int_{\Omega} a(t) f(t) d \mu\right\|+\left(\|a\|_{1}-\left\|\int_{\Omega} \frac{a(t) f(t)}{\|f(t)\|} d \mu\right\|\right) \operatorname{ess} \sup (\|f(\cdot)\|),
\end{aligned}
$$

where $f$ (respectively, $a$ ) is assumed to be an almost everywhere nonzero (respectively, positive) integrable $X$-valued (respectively, real-valued) function on a measure space $(\Omega, \mu)$ with positive measure $\mu$. Obviously, (1.4) (respectively, (1.5)) is a special case of (1.6) (respectively, (1.7)).

Motivated by the results established in [5] (see also [3]), and also by the connection between the Dunkl-Williams inequality and the triangle inequality shown by some authors, in this paper we shall discuss continuous versions of the generalised DunklWilliams inequality in Banach space. We also investigate some applications to infinite series.

\section{Dunkl-Williams inequalities for integrable functions}

Theorem 2.1. Let $X$ be a Banach space, $(\Omega, \mu)$ be a measure space with positive measure $\mu$, and $a(\cdot)$ be an essentially bounded positive integrable function on $\Omega$. If a function $f \in L^{1}(\Omega, X)$ is such that $f(t) \neq 0$ almost everywhere in $\Omega$, then for any fixed $t_{1}, t_{2} \in \Omega$, the following inequalities hold:

$$
\begin{aligned}
& \left\|\int_{\Omega} \frac{a(t) f(t)}{\|f(t)\|} d \mu\right\| \leq \frac{1}{\left\|f\left(t_{1}\right)\right\|}\left(\left\|\int_{\Omega} a(t) f(t) d \mu\right\|+\int_{\Omega}\left|\|f(t)\|-\left\|f\left(t_{1}\right)\right\|\right| a(t) d \mu\right), \\
& \left\|\int_{\Omega} \frac{a(t) f(t)}{\|f(t)\|} d \mu\right\| \geq \frac{1}{\left\|f\left(t_{2}\right)\right\|}\left(\left\|\int_{\Omega} a(t) f(t) d \mu\right\|-\int_{\Omega}\left|\|f(t)\|-\left\|f\left(t_{2}\right)\right\|\right| a(t) d \mu\right) .
\end{aligned}
$$

Proof. Obviously, if $\|f(\cdot)\|$ is constant almost everywhere in $\Omega$, then both inequalities (2.1) and (2.2) hold with equality. Therefore, we may assume this is not 
the case. For (2.1), let us fix any $t_{1} \in \Omega$ such that $f\left(t_{1}\right) \neq 0$. Then we compute:

$$
\begin{aligned}
\left\|\int_{\Omega} \frac{a(t) f(t)}{\|f(t)\|} d \mu\right\| & =\left\|\int_{\Omega} \frac{a(t) f(t)}{\left\|f\left(t_{1}\right)\right\|} d \mu-\int_{\Omega} \frac{a(t) f(t)}{\left\|f\left(t_{1}\right)\right\|} d \mu+\int_{\Omega} \frac{a(t) f(t)}{\|f(t)\|} d \mu\right\| \\
& =\left\|\int_{\Omega} \frac{a(t) f(t)}{\left\|f\left(t_{1}\right)\right\|} d \mu+\int_{\Omega}\left(\frac{1}{\|f(t)\|}-\frac{1}{\left\|f\left(t_{1}\right)\right\|}\right) a(t) f(t) d \mu\right\| \\
& \leq\left\|\int_{\Omega} \frac{a(t) f(t)}{\left\|f\left(t_{1}\right)\right\|} d \mu\right\|+\left\|\int_{\Omega}\left(\frac{1}{\|f(t)\|}-\frac{1}{\left\|f\left(t_{1}\right)\right\|}\right) a(t) f(t) d \mu\right\| \\
& \leq\left\|\int_{\Omega} \frac{a(t) f(t)}{\left\|f\left(t_{1}\right)\right\|} d \mu\right\|+\int_{\Omega} \frac{1}{\|f(t)\|}-\frac{1}{\left\|f\left(t_{1}\right)\right\|} \mid a(t)\|f(t)\| d \mu \\
& =\frac{1}{\left\|f\left(t_{1}\right)\right\|}\left(\left\|\int_{\Omega} a(t) f(t) d \mu\right\|+\int_{\Omega} a(t)\left|\|f(t)\|-\left\|f\left(t_{1}\right)\right\|\right| d \mu\right) .
\end{aligned}
$$

This proves (2.1).

In order to obtain (2.2), we proceed in a similar way for a fixed $t_{2} \in \Omega$ such that $f\left(t_{2}\right) \neq 0$ :

$$
\begin{aligned}
\left\|\int_{\Omega} \frac{a(t) f(t)}{\|f(t)\|} d \mu\right\| & =\left\|\int_{\Omega} \frac{a(t) f(t)}{\left\|f\left(t_{2}\right)\right\|} d \mu-\int_{\Omega} \frac{a(t) f(t)}{\left\|f\left(t_{2}\right)\right\|} d \mu+\int_{\Omega} \frac{a(t) f(t)}{\|f(t)\|} d \mu\right\| \\
& =\left\|\int_{\Omega} \frac{a(t) f(t)}{\left\|f\left(t_{2}\right)\right\|} d \mu-\int_{\Omega}\left(\frac{1}{\left\|f\left(t_{2}\right)\right\|}-\frac{1}{\|f(t)\|}\right) a(t) f(t) d \mu\right\| \\
& \geq\left\|\int_{\Omega} \frac{a(t) f(t)}{\left\|f\left(t_{2}\right)\right\|} d \mu\right\|-\left\|\int_{\Omega}\left(\frac{1}{\left\|f\left(t_{2}\right)\right\|}-\frac{1}{\|f(t)\|}\right) a(t) f(t) d \mu\right\| \\
& \geq\left\|\int_{\Omega} \frac{a(t) f(t)}{\left\|f\left(t_{2}\right)\right\|} d \mu\right\|-\int_{\Omega}\left|\frac{1}{\|f(t)\|}-\frac{1}{\left\|f\left(t_{2}\right)\right\|}\right| a(t)\|f(t)\| d \mu \\
& =\frac{1}{\left\|f\left(t_{2}\right)\right\|}\left(\left\|\int_{\Omega} a(t) f(t) d \mu\right\|-\int_{\Omega}\|f(t)\|-\left\|f\left(t_{2}\right)\right\| \mid a(t) d \mu\right) .
\end{aligned}
$$

Having obtained (2.2), the proof is complete.

REMARK 2.2. The inequalities (1.6) and (1.7) established in [5] can be obtained as a particular case of the results established in Theorem 2.1. We show this as the following corollary.

Corollary 2.3. Let $X$ be a Banach space, $(\Omega, \mu)$ be a measure space with positive measure $\mu$, and $a(\cdot)$ be an essentially bounded positive integrable function on $\Omega$. If a function $f \in L^{1}(\Omega, X)$ is such that $f(t) \neq 0$ almost everywhere in $\Omega$, then (1.6) and (1.7) hold.

Proof. To obtain these results, we follow the proof of [5, Theorem 2.1], but make some modifications. Inequality (1.6) is obvious for the case ess $\inf (\|f(\cdot)\|)=0$. For the case ess $\inf (\|f(\cdot)\|)>0$, take any $t_{1}, t_{2} \in \Omega$ and redefine $f\left(t_{1}\right)$ and $f\left(t_{2}\right)$ as $\left\|f\left(t_{1}\right)\right\|:=$ ess $\sup (\|f(\cdot)\|)\left(\right.$ respectively, $\left.\left\|f\left(t_{2}\right)\right\|:=\operatorname{ess} \inf (\|f(\cdot)\|)\right)$. Then, using (2.1) (respectively, (2.2)) from Theorem 2.1, we obtain (1.6) (respectively, (1.7)). We omit the details. 
Remark 2.4. Actually, (1.6) and (1.7) are equivalent to (2.1) and (2.2) with $\left\|f\left(t_{1}\right)\right\|:=$ ess $\sup (\|f(\cdot)\|)$ and $\left\|f\left(t_{2}\right)\right\|:=\operatorname{ess} \inf (\|f(\cdot)\|)$. But there may be other choices of $f\left(t_{1}\right)$ and $f\left(t_{2}\right)$ which give better estimations for (1.6) and (1.7). In this sense, (2.1) and (2.2) give better estimations than (1.6) and (1.7) obtained by Hsu et al. in [5]. We illustrate this fact in the following example (see [5, Example 2.2]).

ExAmple 2.5. Let $a(t) \equiv 1$ and let $f \in L^{1}\left([-1,1], \mathbb{R}^{2}\right)$ be defined by $f(t)=(t,-1)$ for $t \in[-1,0]$ and $f(t)=(t, 1+t)$ for $t \in(0,1]$. Then $\|f(t)\|_{1}=1-t$ for $t \in[-1,0]$ and $\|f(t)\|_{1}=1+2 t$ for $t \in(0,1]$, and so $\inf \left(\|f(t)\|_{1}\right)=1$ and $\sup \left(\|f(t)\|_{1}\right)=3$.

If we take $t_{1}=t_{2}=-1$, then an elementary calculation shows that (2.1) and (2.2) give $-\frac{1}{4}<0.1633<\frac{3}{4}$.

However, for (2.1), let $\left\|f\left(t_{1}\right)\right\|=\sup \left(\|f(t)\|_{1}\right)=3$; for $(2.2)$, let $\left\|f\left(t_{2}\right)\right\|=$ $\inf \left(\|f(t)\|_{1}\right)=1$. Then

$$
1 \times\left(\frac{1}{2}-\frac{3}{2}\right)(=-1)<0.1633<\frac{1}{3} \times\left(\frac{1}{2}+\frac{5}{2}\right)(=1) .
$$

So we can choose some $t_{1}, t_{2} \in[-1,1]$ such that the results (2.1) and (2.2) give better estimates than those in (1.6) and (1.7). Hence, Theorem 2.1 is more meaningful.

\section{Attaining equality in a strictly convex Banach space}

In what follows we shall consider the attaining of equality for each of our inequalities in a strictly convex Banach space. Proposition 2.3 of [5] is quite useful for our subsequent discussions. In order to cite it conveniently, we list some results as the following lemma.

Lemma 3.1. Let $X$ be a strictly convex Banach space and $(\Omega, \mu)$ be a measure space with positive measure $\mu$, and let $f \in L^{1}(\Omega, X)$ such that $f(t) \neq 0$ almost everywhere in $\Omega$. Then the following statements are equivalent:

(a) $\left\|\int_{\Omega} f(t) d \mu\right\|=\int_{\Omega}\|f(t)\| d \mu$;

(b) $\quad\left\|\int_{\Omega}|h(t)| f(t) d \mu\right\|=\int_{\Omega}|h(t)|\|f(t)\| d \mu$ for some essentially bounded measurable function $h:(\Omega, \mu) \rightarrow(-\infty, \infty)$;

(c) $\quad f(t) /\|f(t)\|$ is a constant unit vector almost everywhere in $\Omega$.

The following theorem gives conditions for equality in (2.1) to hold in a strictly convex Banach space.

THeOREM 3.2. Let $X$ be a strictly convex Banach space, $(\Omega, \mu)$ be a measure space with positive measure $\mu$, and $a(\cdot)$ be an essentially bounded positive integrable function on $\Omega$. If a function $f \in L^{1}(\Omega, X)$ is such that $f(t) \neq 0$ almost everywhere in $\Omega$, then, for any fixed $t_{1} \in \Omega$,

$$
\left\|\int_{\Omega} \frac{a(t) f(t)}{\|f(t)\|} d \mu\right\|=\frac{1}{\left\|f\left(t_{1}\right)\right\|}\left(\left\|\int_{\Omega} a(t) f(t) d \mu\right\|+\int_{\Omega}\left|\|f(t)\|-\left\|f\left(t_{1}\right)\right\|\right| a(t) d \mu\right)
$$


if and only if one of the following two conditions holds:

(a) $\quad\|f(t)\|$ is constant almost everywhere in $\Omega$;

(b) $\quad f(t) /\|f(t)\|$ is a constant unit vector almost everywhere in $\Omega$.

Proof. We first note that, according to (2.1) in the proof of Theorem 2.1, (3.1) holds if and only if the following two conditions are satisfied:

$$
\left\|\int_{\Omega} \frac{a(t) f(t)}{\|f(t)\|} d \mu\right\|=\left\|\int_{\Omega} \frac{a(t) f(t)}{\left\|f\left(t_{1}\right)\right\|} d \mu\right\|+\left\|\int_{\Omega}\left(\frac{1}{\|f(t)\|}-\frac{1}{\left\|f\left(t_{1}\right)\right\|}\right) a(t) f(t) d \mu\right\|,
$$

and

$$
\left\|\int_{\Omega}\left(\frac{1}{\|f(t)\|}-\frac{1}{\left\|f\left(t_{1}\right)\right\|}\right) a(t) f(t) d \mu\right\|=\int_{\Omega}\left|\frac{1}{\|f(t)\|}-\frac{1}{\left\|f\left(t_{1}\right)\right\|}\right| a(t)\|f(t)\| d \mu .
$$

Let (3.1) hold true and assume that the assertion (a) is not the case. Then, by (3.3) and Lemma 3.1, we can prove that $f(t) /\|f(t)\|$ is a constant unit vector almost everywhere in $\Omega$.

Conversely, if $\|f(t)\|$ is constant almost everywhere in $\Omega$, then (3.1) holds trivially. So we assume that this is not the case. Therefore, we assume case (b). Note that (3.1) is equivalent to (3.2) and (3.3). Thus, in order to prove (3.1), we must show that (3.2) and (3.3) hold. Since $f(t) /\|f(t)\|$ is a constant unit vector almost everywhere in $\Omega$, then by Lemma 3.1, noting that $X$ is strictly convex, conditions (3.2) and (3.3) are clearly satisfied. This completes the proof.

The next theorem gives conditions for equality in (2.2) to hold in a strictly convex normed linear space. The proof is similar to that of Theorem 3.2, and we omit it.

Theorem 3.3. Let $X$ be a a strictly convex Banach space, $(\Omega, \mu)$ be a measure space with positive measure $\mu$, and $a(\cdot)$ be an essentially bounded positive integrable function on $\Omega$. If a function $f \in L^{1}(\Omega, X)$ is such that $f(t) \neq 0$ almost everywhere in $\Omega$, then, for any fixed $t_{2} \in \Omega$,

$$
\left\|\int_{\Omega} \frac{a(t) f(t)}{\|f(t)\|} d \mu\right\|=\frac{1}{\left\|f\left(t_{2}\right)\right\|}\left(\left\|\int_{\Omega} a(t) f(t) d \mu\right\|-\int_{\Omega} a(t)\left|\|f(t)\|-\left\|f\left(t_{2}\right)\right\|\right| d \mu\right)
$$

if and only if one of the following two conditions holds:

(a) $\quad\|f(t)\|$ is constant almost everywhere in $\Omega$;

(b) $\quad f(t) /\|f(t)\|$ is a constant unit vector almost everywhere in $\Omega$.

\section{Application to infinite series}

For discrete versions of the results in Section 2 , by letting $\Omega=\mathbb{N}, \mu(n):=1$ and $a(n):=a_{n}$ for $n \in \mathbb{N}$ in Theorem 2.1, we can obtain results about the generalised Dunkl-Williams inequality and its reverse for infinite series as the following theorem. 
THEOREM 4.1. Let $\left\{a_{n}\right\}$ be a sequence of nonnegative numbers such that $\sum_{n=1}^{\infty} a_{n}<\infty$. Then, for any sequence $\left\{x_{n}\right\}$ of nonzero elements in a normed linear space $X$ such that $\sum_{n=1}^{\infty} a_{n}\left\|x_{n}\right\|<\infty$,

$$
\begin{aligned}
& \left\|\sum_{j=1}^{\infty} a_{j} \frac{x_{j}}{\left\|x_{j}\right\|}\right\| \leq \inf _{i}\left\{\frac{1}{\left\|x_{i}\right\|}\left(\left\|\sum_{j=1}^{\infty} a_{j} x_{j}\right\|+\sum_{j=1}^{\infty} a_{j} \mid\left\|x_{j}\right\|-\left\|x_{i}\right\|\right)\right\}, \\
& \left\|\sum_{j=1}^{\infty} a_{j} \frac{x_{j}}{\left\|x_{j}\right\|}\right\| \geq \sup _{i}\left\{\frac{1}{\left\|x_{i}\right\|}\left(\left\|\sum_{j=1}^{\infty} a_{j} x_{j}\right\|-\sum_{j=1}^{\infty} a_{j} \mid\left\|x_{j}\right\|-\left\|x_{i}\right\|\right)\right\} .
\end{aligned}
$$

\section{Acknowledgement}

The author would like to thank the referee for useful comments which greatly improved the presentation of this paper.

\section{References}

[1] F. Dadipour, M. S. Moslehian, J. M. Rassias and S.-E. Takahasi, 'Characterization of a generalized triangle inequality in normed spaces', Nonlinear Anal. 75(2) (2012), 735-741.

[2] S. S. Dragomir, 'Reverses of the triangle inequality in Banach spaces', J. Inequal. Pure Appl. Math. 6(5) (2005), 46; Art. 129.

[3] S. S. Dragomir, 'Reverses of the continuous triangle inequality for Bochner integral in complex Hilbert spaces', J. Math. Anal. Appl. 329(1) (2007), 65-76.

[4] C. F. Dunkl and K. S. Williams, 'A simple norm inequality', Amer. Math. Monthly. 71 (1964), 53-54.

[5] C.-Y. Hsu, S.-Y. Shaw and H.-J. Wong, 'Refinements of generalized triangle inequalities', J. Math. Anal. Appl. 344 (2008), 613-639.

[6] M. Kato, K.-S. Saito and T. Tamura, 'Sharp triangle inequality and its reverse in Banach space', Math. Inequal. Appl. (2007), 451-460.

[7] L. Maligranda, 'Simple norm inequalities', Amer. Math. Monthly. 133 (2006), 256-260.

[8] P. R. Mercer, 'The Dunkl-Williams inequality in an inner-product space', Math. Inequal. Appl. 10 (2007), 447-451.

[9] J. Pečarič and R. Rajič, 'The Dunkl-Williams inequality with $n$ elements in normed linear spaces', Math. Inequal. Appl. 10 (2007), 461-470.

[10] J. Pečarič and R. Rajič, 'The Dunkl-Williams equality in pre-Hilbert $C^{*}$-modules', Linear. Algebra. Appl. 425 (2007), 16-25.

[11] S.-E. Takahasi, J. M. Rassias, S. Saitoh and Y. Takahashi, 'Refined generalizations of the triangle inequality on Banach spaces', Math. Inequal. Appl. 13(4) (2010), 733-741.

JIANBING CAO, Department of Mathematics, East China Normal University, Dongchuan RD 500, Shanghai 200241, PR China

and

Department of Mathematics, Henan Institute of Science and Technology,

Xinxiang, Henan 453003, PR China

e-mail: caocjb@163.com 